\title{
Time-varying impedance of the sheath on a probe in an $\mathrm{RF}$ plasma
}

\author{
Francis F Chen \\ Electrical Engineering Department, University of California, Los Angeles, 90095-1594, USA
}

Received 29 November 2005, in final form 30 November 2005

Published 29 August 2006

Online at stacks.iop.org/PSST/15/773

\begin{abstract}
Langmuir probes used in radiofrequency (rf) discharges usually include compensation elements that minimize the effect of high frequency oscillations in plasma potential. The design of these elements requires knowledge of the capacitance of the sheath on the probe tip, a quantity which varies nonlinearly during the $\mathrm{rf}$ cycle. Sheath capacitance has been studied previously for capacitively coupled discharges, where the rf is applied to the electrodes. Here the problem is treated from the standpoint of a small probe in a fluctuating discharge. This work differs from existing literature in that (a) no step model is used and the Debye sheath is treated exactly, (b) the treatment is simple and analytic, (c) the time-variation of the capacitance is explicitly shown, (d) the results are applied to probe design and (e) cylindrical geometry is considered. The rf frequency is assumed low enough that electron transit times can be ignored. We find that when the rf excursions bring the sheath from the Child-Langmuir region into the Debye sheath or electron saturation region, its capacitance has a strongly non-linear behaviour.
\end{abstract}

(Some figures in this article are in colour only in the electronic version)

\section{Introduction}

In the design of rf-compensated Langmuir probes for measurements in rf plasmas, it is necessary to know the capacitive coupling through the sheath of rf fluctuations in plasma potential. The simple approach normally used is to consider the sheath to be a vacuum capacitor whose thickness is roughly estimated. In plane geometry this thickness is not well defined, even if a sheath edge is well defined, because the thickness depends on the slope of the potential at the edge, and this depends on the transition to the presheath. It is impractical to solve for the presheath, since the solution depends on collisions and ionization and is specific to each discharge. More accurate treatments of rf sheaths can be found in the literature but are not always suitable for the present task.

Lieberman [1,2] has given analytic solutions for the sheath on a driven electrode in a capacitively coupled plasma (CCP). However, he used a model in which the electron density was approximated by a single step. Godyak and Sternberg [3] pointed out that high-and low-frequency approximations can be made depending on whether the rf frequency $\omega$ is larger or smaller than the ion plasma frequency $\Omega_{\mathrm{p}}$, and they solved [4] the high-frequency case for a CCP driven symmetrically relative to ground. How the shape of the sheath changes during the rf cycle was computed numerically by Zhang et al [5], with the result that large changes occur in the low-frequency case, the one treated in this paper. However, they did not give the sheath capacitance explicitly.

Godyak [3] showed that the sheath capacitance $C_{\mathrm{sh}}$ depends only on the surface charge on the probe and can be calculated without solving for the sheath thickness numerically. Sudit and Chen [6] used this shortcut to calculate $C_{\text {sh }}$. In that work, however, they neglected the Debye sheath, treating only the Child-Langmuir $(\mathrm{C}-\mathrm{L})$ sheath, adding, rather inconsistently, the Bohm velocity at the sheath edge. Here we solve the plane sheath problem consistently, showing exactly what approximations were previously made and also obtaining formulae from which the sheath capacitance can be calculated even when the probe is not biased far from the space potential. To establish notation, we start with a brief review of plane sheath theory before applying it to the calculation of sheath capacitance as a function of time. Cylindrical sheaths and the resistive part of the sheath impedance will be treated at the end. 


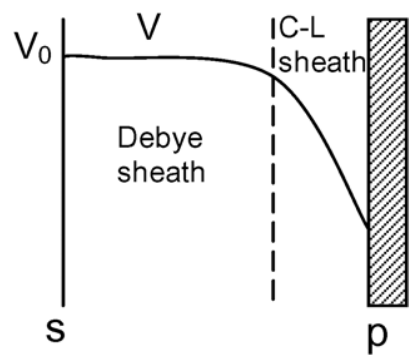

Figure 1. Geometry of a plane sheath.

\section{Plane sheaths in a nutshell}

\subsection{Basic equations}

We start by defining a sheath edge s (figure 1) with $V_{\mathrm{s}}, n_{\mathrm{s}}$ and $v_{\mathrm{s}}$ denoting, respectively, the potential, density and ion velocity there. Following traditional practice, we set $V_{\mathrm{s}}=0$ in the absence of $\mathrm{rf}$ and assume quasineutrality up to $\mathrm{s}$ so that $n_{\mathrm{i}}(s)=n_{\mathrm{e}}(s) \equiv n_{\mathrm{s}}$. The ions enter the sheath with a unidirectional, monoenergetic velocity $v_{\mathrm{s}}$, whose Bohm value will be recovered in due course. There is no artificial separation between the Debye sheath (where $n_{\mathrm{e}} \neq 0$ ) and the ChildLangmuir sheath (where $n_{\mathrm{e}}=0$ ). Defining

$$
V=\tilde{V}-V_{\mathrm{s}},
$$

where $\tilde{V}$ is the actual potential as $V_{\mathrm{s}}$ varies, we write Poisson's equation as

$$
\varepsilon_{0} \frac{\mathrm{d}^{2} V}{\mathrm{~d} x^{2}}=e\left(n_{\mathrm{e}}-n_{\mathrm{i}}\right) .
$$

For Maxwellian electrons, we have

$$
n_{\mathrm{e}}=n_{\mathrm{s}} \mathrm{e}^{e V / K T_{\mathrm{e}}} .
$$

The ion velocity $v$ is given by energy conservation

$$
\frac{1}{2} M v_{\mathrm{s}}^{2}=\frac{1}{2} M v^{2}+e V(V \leqslant 0)
$$

so that

$$
v=\left(v_{\mathrm{s}}^{2}-\frac{2 e V}{M}\right)^{1 / 2} .
$$

Since ion flux is conserved, we have

$$
n_{\mathrm{i}} v_{\mathrm{i}}=n_{\mathrm{s}} v_{\mathrm{s}}, \quad n_{\mathrm{i}}=n_{\mathrm{s}} v_{\mathrm{s}} / v_{\mathrm{i}}=n_{\mathrm{s}}\left(1-\frac{2 e V}{M v_{\mathrm{s}}^{2}}\right)^{-1 / 2} .
$$

The positive, dimensionless potential $\eta$ is defined as

$$
\eta \equiv-e\left(\tilde{V}-V_{\mathrm{s}}\right) / K T_{\mathrm{e}}=-e V / K T_{\mathrm{e}}
$$

whereupon Poisson's equation becomes

$$
\frac{\varepsilon_{0} K T_{\mathrm{e}}}{n_{\mathrm{s}} e^{2}} \frac{\mathrm{d}^{2} \eta}{\mathrm{d} x^{2}}=\left(1+\frac{2 K T_{\mathrm{e}}}{M v_{\mathrm{s}}^{2}} \eta\right)^{-1 / 2}-\mathrm{e}^{-\eta}
$$

Henceforth we use a Roman 'e' for 2.718 and an italic ' $e$ ' for charge. Normalizing $x$ to the Debye length (with $n=n_{\mathrm{s}}$ ),

$$
\lambda_{\mathrm{D}}=\left(\varepsilon_{0} K T_{\mathrm{e}} / n_{\mathrm{s}} e^{2}\right)^{1 / 2}, \quad \xi \equiv(x-s) / \lambda_{\mathrm{D}}
$$

and defining the ion acoustic speed $c_{\mathrm{s}}$ and the Mach number $\mathcal{M}$ as

$$
c_{\mathrm{s}} \equiv\left(K T_{\mathrm{e}} / M\right)^{1 / 2}, \quad \mathcal{M} \equiv v_{\mathrm{s}} / c_{\mathrm{s}},
$$

equation (8) becomes simply

$$
\eta^{\prime \prime}=\frac{\mathrm{d}^{2} \eta}{\mathrm{d} \xi^{2}}=\left(1+2 \eta / \mathcal{M}^{2}\right)^{-1 / 2}-\mathrm{e}^{-\eta} .
$$

Multiplying by the integrating factor $\eta^{\prime}$ and integrating from $\xi=0$, we obtain

$$
\frac{1}{2}\left(\eta^{\prime}\right)^{2}=\mathcal{M}^{2}\left[\left(1+2 \eta / \mathcal{M}^{2}\right)^{1 / 2}-1\right]+\left[\mathrm{e}^{-\eta}-1\right] .
$$

Here we have used the sheath boundary condition $\eta^{\prime}(0)=0$.

\subsection{Recovery of the Bohm sheath criterion}

Since equation (12) has to be positive for all $\eta$, we can get a condition on $\mathcal{M}$ by expanding the rhs for small $\eta$, up to order $\eta^{2}$.

$$
\begin{aligned}
& \frac{1}{2}\left(\eta^{\prime}\right)^{2}=\mathcal{M}^{2}\left[\left(1+\eta / \mathcal{M}^{2}-\frac{1}{2} \eta^{2} / \mathcal{M}^{4}\right)-1\right] \\
& \quad+\left[1-\eta+\frac{1}{2} \eta^{2}-1\right]=-\frac{1}{2} \eta^{2} / \mathcal{M}^{2}+\frac{1}{2} \eta^{2} \geqslant 0 .
\end{aligned}
$$

Hence, $\mathcal{M} \geqslant 1$, or $v_{\mathrm{s}} \geqslant c_{\mathrm{s}}$, which is the Bohm criterion. We can now define the 'sheath edge' to be that position s near the wall or probe where this condition is barely satisfied: $v_{\mathrm{s}}=c_{\mathrm{s}}$. Setting $\mathcal{M}=1$ in equation (12) and taking the square root, we obtain

$$
\eta^{\prime}= \pm \sqrt{2}\left[(1+2 \eta)^{1 / 2}+\mathrm{e}^{-\eta}-2\right]^{1 / 2} .
$$

This equation differs from previous work on $C_{\mathrm{sh}}$ in that the electron density is not neglected or approximated.

\subsection{Recovery of the Child-Langmuir law}

For space-charge-limited ion emission, the electron terms are omitted, and $\eta$ is infinitely large, since temperatures are zero. Equation (14) then becomes

$$
\eta^{\prime}=2^{1 / 2}(2 \eta)^{1 / 4}, \quad \eta^{-1 / 4} \eta^{\prime}=2^{3 / 4} .
$$

Integrating from $\xi=0$ to $d / \lambda_{D}$ gives

$$
\eta^{3 / 4}=\frac{3}{4} 2^{3 / 4} \xi_{\mathrm{d}}, \quad \eta^{3 / 2}=\frac{9}{16} 2^{3 / 2} \xi_{\mathrm{d}}^{2}=\frac{9}{8} 2^{1 / 2} \xi_{\mathrm{d}}^{2} .
$$

Converting back to dimensional units, we have

$$
\left(\frac{-e V}{K T_{\mathrm{e}}}\right)^{3 / 2}=\frac{9}{8} 2^{1 / 2} d^{2}\left(\frac{n_{\mathrm{s}} e^{2}}{\varepsilon_{0} K T_{\mathrm{e}}}\right) .
$$

It is now convenient to express $n_{\mathrm{s}}$ in terms of the ion current density $J$ :

$$
J=e n_{\mathrm{s}} c_{\mathrm{s}}=e n_{\mathrm{s}}\left(K T_{\mathrm{e}} / M\right)^{1 / 2}, \quad n_{\mathrm{s}}=J / e\left(K T_{\mathrm{e}} / M\right)^{1 / 2} .
$$

The normalizing factor $K T_{\mathrm{e}}$ now cancels out, as it should, and we have

$$
J=\frac{4}{9}\left(\frac{2 e}{M}\right)^{1 / 2} \frac{\varepsilon_{0}(-V)^{3 / 2}}{d^{2}},
$$




\subsection{Relation to the plasma potential}

Since the ions have a velocity $c_{\mathrm{s}}$ at $s$, they must have gained an energy $\frac{1}{2} M c_{\mathrm{s}}^{2}=\frac{1}{2} K T_{\mathrm{e}}$ in the presheath, so that the potential $V_{0}$ in the main plasma must be higher than $V_{\mathrm{s}}$ by $\frac{1}{2} K T_{\mathrm{e}} / e$. The electrons, if Maxwellian, would have a density higher by a factor $e^{1 / 2}$ in the plasma than at the sheath edge. Thus, $n_{\mathrm{s}}=\mathrm{e}^{-1 / 2} n_{0}=0.61 n_{0}$. Since $\lambda_{\mathrm{D}}$ was defined with $n_{\mathrm{s}}^{-1 / 2}$, its value would be decreased by $\mathrm{e}^{-1 / 4}=0.78$ if we had chosen to define it using $n_{0}$. The derivative $\eta^{\prime}$ would be increased by a factor $e^{1 / 4}=1.28$. However, once this is understood, it is not necessary to make any changes in the formulism.

\section{Calculation of sheath capacitance}

The charge on a capacitor is given by $Q=C V$. From this, the sheath capacitance $C_{\mathrm{sh}}$ can be written as

$$
\frac{C_{\mathrm{sh}}}{A_{\mathrm{p}}}=\frac{\Delta \rho_{\mathrm{s}}}{\Delta V}
$$

where $A_{\mathrm{p}}$ is the probe area and $\rho_{\mathrm{s}}$ is the surface charge density on the probe. Following [6], we use Gauss's Law to obtain $\rho_{\mathrm{s}}=D_{\mathrm{n}}$, the normal component of $\varepsilon_{0} E=-\varepsilon_{0}(-\nabla V)$. The first minus sign comes from the fact that $D_{\mathrm{n}}$ is defined in the $-x$ direction in figure 1. Equation (20) then becomes

$$
\frac{C_{\mathrm{sh}}}{A_{\mathrm{p}}}=-\varepsilon_{0} \frac{\Delta E}{\Delta V}=\varepsilon_{0} \frac{\mathrm{d}}{\mathrm{d} V}\left(\frac{\mathrm{d} V}{\mathrm{~d} x}\right)=\frac{\varepsilon_{0}}{\lambda_{\mathrm{D}}} \frac{\mathrm{d}}{\mathrm{d} \eta}\left(\frac{\mathrm{d} \eta}{\mathrm{d} \xi}\right) .
$$

Equation (14) gives $\mathrm{d} \eta / \mathrm{d} \xi$. Taking its $\eta$-derivative, we obtain

$$
\frac{C_{\mathrm{sh}}}{A_{\mathrm{p}}}=\frac{\varepsilon_{0}}{\lambda_{\mathrm{D}}} \frac{1}{\sqrt{2}} \frac{(1+2 \eta)^{\frac{1}{2}}-\mathrm{e}^{-\eta}}{\left[(1+2 \eta)^{\frac{1}{2}}+\mathrm{e}^{-\eta}-2\right]^{1 / 2}},
$$

where we have taken the + sign because the ion density (the first term in the numerator) has to be larger than the electron density (the second term) once the Bohm criterion is satisfied. Here it is understood that $\eta$ is evaluated at the probe, so that $\eta=-e\left(V_{\mathrm{p}}-V_{\mathrm{s}}\right) / K T_{\mathrm{e}}$. This equation is valid for all $V_{\mathrm{p}}$ below the space potential even if $\eta$ is small.

The error in neglecting the Debye sheath [6] can now be calculated. If we neglect $\mathrm{e}^{-\eta}$ and its integration constant, the fraction $F$ in equation (22) becomes

$F=(1+2 \eta)^{-\frac{1}{2}}\left[(1+2 \eta)^{\frac{1}{2}}-1\right]^{-\frac{1}{2}}=\left[(1+2 \eta)^{3 / 2}-(1+2 \eta)\right]^{-\frac{1}{2}}$.

This can be expanded in the small quantity $\varepsilon \equiv 1 /(2 \eta)$ to obtain

$$
\begin{aligned}
F= & \varepsilon^{3 / 4}\left(1-\varepsilon^{1 / 2}+\frac{3}{2} \varepsilon-\cdots\right)^{-1 / 2} \\
& \approx \varepsilon^{3 / 4}\left(1+\frac{1}{2} \varepsilon^{1 / 2}-\frac{3}{4} \varepsilon+\cdots\right) .
\end{aligned}
$$

Thus the capacitance is approximately

$$
\frac{C_{\mathrm{sh}}}{A_{\mathrm{p}}}=\frac{\varepsilon_{0}}{\lambda_{\mathrm{D}}} \frac{(2 \eta)^{-3 / 4}}{2^{\frac{1}{2}}}\left(1+\frac{1}{2} \frac{1}{(2 \eta)^{\frac{1}{2}}}\right) .
$$

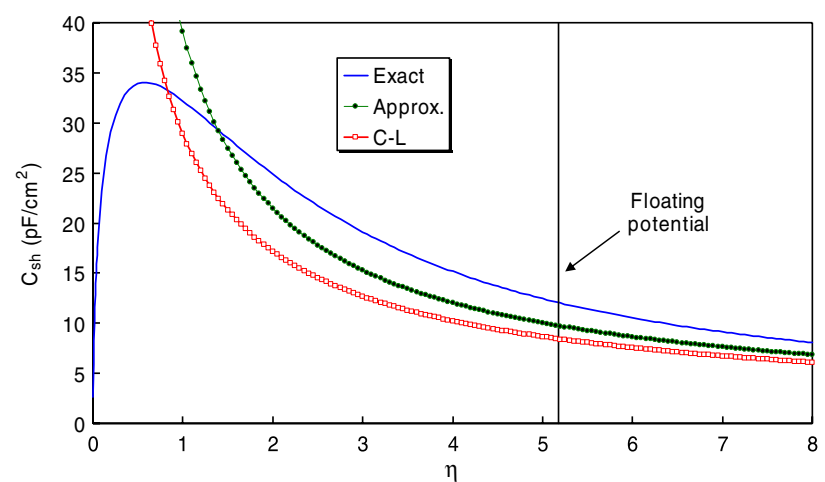

Figure 2. Sheath capacitance versus normalized probe bias according to the exact formula (-), its Taylor expansion $(\bullet)$ and the Child-Langmuir formula $(\mathrm{O})$.

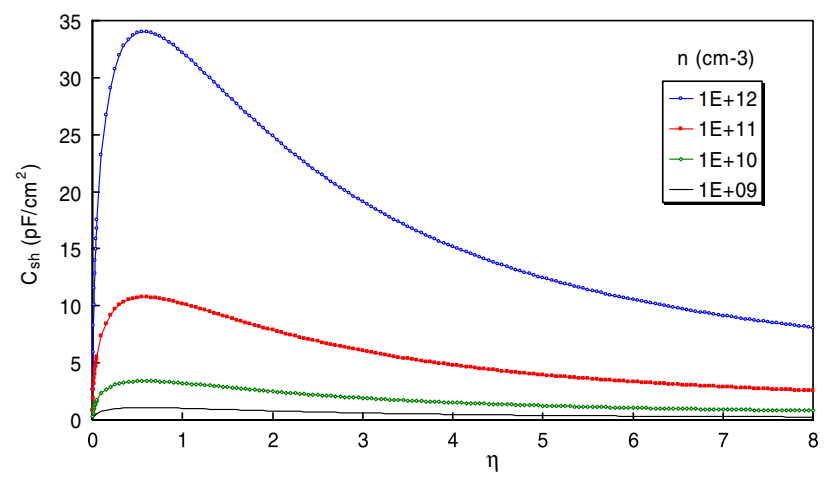

Figure 3. Variation of dc $C_{\mathrm{sh}}$ with density.

Apart from notation, this is the same as equation (17) of [6] if one approximates $n_{\mathrm{s}}$ with $\frac{1}{2} n_{0}$ (section 2.4). The correction term in equation (25), which modifies the Child-Langmuir sheath for the incident ion velocity, is not small. At the floating potential, $\eta$ is about 5 , so that term is $\approx 0.16$. For smaller $\eta\left(V_{\mathrm{p}}\right.$ closer to space potential) the expansion fails, and one should use the exact equation (22), which includes the Debye sheath. Physically, equation (25) tells us that the sheath capacitance increases as $\eta$ decreases - that is, as the sheath gets thinnermore or less as predicted by the Child-Langmuir law. There is, in addition, a dependence on $T_{\mathrm{e}}$ and $n_{0}$ through $\lambda_{\mathrm{D}}$.

Figure 2 shows computations of $C_{\mathrm{sh}}$ versus $\eta$ comparing the exact formula of equation (22) with the approximate formulae of equations (25) and (28). At large $\eta$, all the curves show a decrease in $C_{\text {sh }}$ with $\eta$ as the $\mathrm{C}-\mathrm{L}$ sheath thickness increases. The exact curve shows a peak at small $\eta$ as the probe enters the Debye sheath, and it falls at smaller $\eta$ as the thickness of the Debye sheath increases to $\infty$ as $V_{\mathrm{p}}$ reaches the space potential. Approximations that do not include the Debye sheath do not have this feature. The approximation (25) that includes the ion velocity at the sheath edge is somewhat better than the $\mathrm{C}-\mathrm{L}$ approximation (28), which does not. The improvement is not great, since the series in equation (24) converges very slowly with $\eta^{\frac{1}{2}}$. At the floating potential either approximation is reasonably good, but they fail when the $V_{\mathrm{p}}$ moves closer to $V_{\mathrm{s}}$. Figure 3 shows the expected variation of $C_{\text {sh }}$ with density. 


\section{Effect of RF}

\subsection{Range of validity}

In rf discharges, an electric field is applied to the plasma either by an electrode or by an external antenna. This E-field can drive electrons towards a wall. The sheath drop there must then increase to repel enough electrons to maintain a neutral plasma, and the plasma potential $V_{0}$ must rise. As the $\mathrm{rf}$ changes sign, $V_{0}$ will rise and fall unsymmetrically, since the current through a Coulomb barrier varies exponentially with voltage. The harmonic content of $V_{0}$ oscillations will vary in different devices. Godyak and Piejak [7] showed that the 2nd harmonic will dominate in a CCP with electrodes driven symmetrically relative to ground [4]. In a normal CCP with one electrode near ground, the fundamental rf frequency $\omega$ will dominate. In a cylindrical inductively coupled plasma (ICP), the E-field is ideally everywhere parallel to the walls so that no oscillating wall sheaths develop, but asymmetries in antenna construction, ports on the walls and capacitive coupling can cause $V_{0}$ oscillations at the fundamental frequency $\omega$. In helicon discharges, an E-field at $\omega$ is an intrinsic property of the helicon wave. To simplify the problem, we will treat one frequency at a time and assume that $V_{\mathrm{s}}$, the sheath-edge potential, follows the oscillations in $V_{0}$.

The dc sheath theory given above is applicable only if the sheath comes into equilibrium at each phase of the rf. This requires, first, that the $\mathrm{rf}$ frequency $f_{\mathrm{rf}}$ be low enough that the electrons respond instantaneously; this is a good assumption. Second, the frequency must be low enough that the ions traverse the sheath before it changes, and their rf motion need not be taken into account $[3,5]$. If the sheath thickness is about $5 \lambda_{\mathrm{D}}$, the ion transit time $t$ through the sheath is

$$
t \approx 5 \lambda_{\mathrm{D}} / c_{\mathrm{s}} \approx 5\left(\frac{\varepsilon_{0} K T_{\mathrm{e}}}{n_{0} e^{2}}\right)^{1 / 2}\left(\frac{M}{K T_{\mathrm{e}}}\right)^{1 / 2}=\frac{5}{\Omega_{\mathrm{p}}},
$$

$\Omega_{\mathrm{p}}$ being the ion plasma frequency. The $\mathrm{rf}$ period $\tau=1 / f_{\mathrm{rf}}=$ must then be $\gg 2 t$, yielding

$$
f_{\text {rf }} \ll \Omega_{\mathrm{p}} / 10 .
$$

At $n_{0}=10^{12} \mathrm{~cm}^{-3}, \Omega_{\mathrm{p}}$ is $\approx 2 \times 10^{8} \mathrm{~s}^{-1}$, so that $f_{\mathrm{rf}}$ must be $\ll 20 \mathrm{MHz}$. This is marginally acceptable at $13.56 \mathrm{MHz}$, and the condition is not met at lower densities even at that frequency. Fortunately, the value of $C_{\mathrm{sh}}$ need not be known exactly in practical applications.

This being the case, we may use equation (25) without the correction term; the error entailed was discussed in section 3. In dimensional units, this is

$$
C_{\mathrm{sh}}=\frac{A_{\mathrm{p}}}{2^{5 / 4}} \frac{\varepsilon_{0}}{\lambda_{\mathrm{D}}}\left[\frac{e\left(V_{\mathrm{s}}-V_{\mathrm{p}}\right)}{K T_{\mathrm{e}}}\right]^{-3 / 4} .
$$

In the absence of $\mathrm{rf}$, the capacitance is [6]

$$
C_{0}=\frac{A_{\mathrm{p}}}{2^{5 / 4}} \frac{\varepsilon_{0}}{\lambda_{\mathrm{D}}}\left[\frac{e\left(\bar{V}_{\mathrm{s}}-\bar{V}_{\mathrm{p}}\right)}{K T_{\mathrm{e}}}\right]^{-3 / 4},
$$

where the overbar denotes the dc values.

\subsection{Uncompensated probe}

4.2.1. Small rf fluctuations. In the presence of $\mathrm{rf}$, consider first a probe or small electrode connected directly to a dc power supply, giving $V_{\mathrm{p}}=\bar{V}_{\mathrm{p}}$. Let $V_{\mathrm{s}}$ oscillate at frequency $\omega$ :

$$
V_{\mathrm{s}}=\bar{V}_{\mathrm{s}}+V_{\mathrm{rf}} \sin \omega t .
$$

The capacitance is then given by

$$
C_{\mathrm{sh}}=C_{0}\left(1+\frac{V_{\mathrm{rf}} \sin \omega t}{\bar{V}_{\mathrm{s}}-\bar{V}_{\mathrm{p}}}\right)^{-3 / 4} .
$$

In addition to $C_{\mathrm{sh}}$ changing with probe voltage, it also changes during an rf cycle. If $V_{\mathrm{rf}}$ is small, equation (31) can be expanded and averaged over an rf cycle. We then find that $\left\langle C_{\mathrm{sh}}\right\rangle$ differs from $C_{0}$ only in second order in $V_{\mathrm{rf}} /\left(\bar{V}_{\mathrm{s}}-\bar{V}_{\mathrm{p}}\right)$.

4.2.2. Large $r f$ fluctuations. A more likely situation, however, is that $V_{\text {rf }}$ is larger than $\bar{V}_{\mathrm{s}}-\bar{V}_{\mathrm{p}}$, which can be as small as $2 K T_{\mathrm{e}} / e$ as the probe $I-V$ curve is swept, while $V_{\mathrm{rf}}$ can exceed $100 \mathrm{~V}$. In that case, equation (31) shows that $C_{\mathrm{sh}}$ has a pole and becomes complex when the bracketed quantity goes negative. This cannot happen physically, however. When $V_{\mathrm{s}}$ comes close to $V_{\mathrm{p}}$, the probe draws a large electron current, raising the mean plasma potential $\bar{V}_{\mathrm{s}}$. Thus, $\bar{V}_{\mathrm{s}}$ has to increase, keeping the bracket positive. Since $\bar{V}_{\mathrm{s}}$ is no longer constant and the approximation $\eta \gg 1$ is not always valid, we must use the exact equation (22):

$$
\frac{C_{\mathrm{sh}}}{A_{\mathrm{p}}}=\frac{\varepsilon_{0}}{\lambda_{\mathrm{D}}} \frac{1}{\sqrt{2}} \frac{(1+2 \eta)^{-\frac{1}{2}}-\mathrm{e}^{-\eta}}{\left[(1+2 \eta)^{\frac{1}{2}}+\mathrm{e}^{-\eta}-2\right]^{1 / 2}} .
$$

Under normal circumstances, $\eta$ is always positive if the probe is biased below $V_{\mathrm{s}}$. The denominator is positive if the Bohm criterion is satisfied (section 2.2), and the numerator is always positive since its leading term in a Taylor expansion is $\eta^{2}$. Recall that $\eta$ is defined as

$$
\eta=\frac{e}{K T_{\mathrm{e}}}\left[V_{\mathrm{rf}} \sin \omega t+\left(\bar{V}_{\mathrm{s}}-\bar{V}_{\mathrm{p}}\right)\right] .
$$

If $\sin \omega t$ is positive or only slightly negative so that $\eta$ remains positive, electron saturation is never reached, and equation (32) is still correct. For more negative values of $\sin \omega t, \eta$ would become negative unless $\bar{V}_{\mathrm{s}}$ increases. The amount of this increase depends on the geometry. To keep the plasma neutral, the electron flux to the probe cannot exceed the ion flux to the walls. For simplicity, we neglect the ion flux to the probe and the electron flux to the walls. The electron flux to the probe is

$$
J_{\mathrm{e}}=A_{\mathrm{p}} n_{0} v_{\mathrm{r}} \mathrm{e}^{-\eta} \quad(\eta \geqslant 0), \quad v_{\mathrm{r}}=\left(K T_{\mathrm{e}} / 2 \pi m\right)^{\frac{1}{2}},
$$

where $v_{\mathrm{r}}$ is the electrons' random thermal velocity. The ion flux to the walls of area $A_{\mathrm{w}}$ is

$$
J_{\mathrm{i}}=A_{\mathrm{w}} n_{\mathrm{s}} c_{\mathrm{s}}=A_{\mathrm{w}} \mathrm{e}^{-\frac{1}{2}} n_{0}\left(K T_{\mathrm{e}} / M\right)^{\frac{1}{2}} .
$$



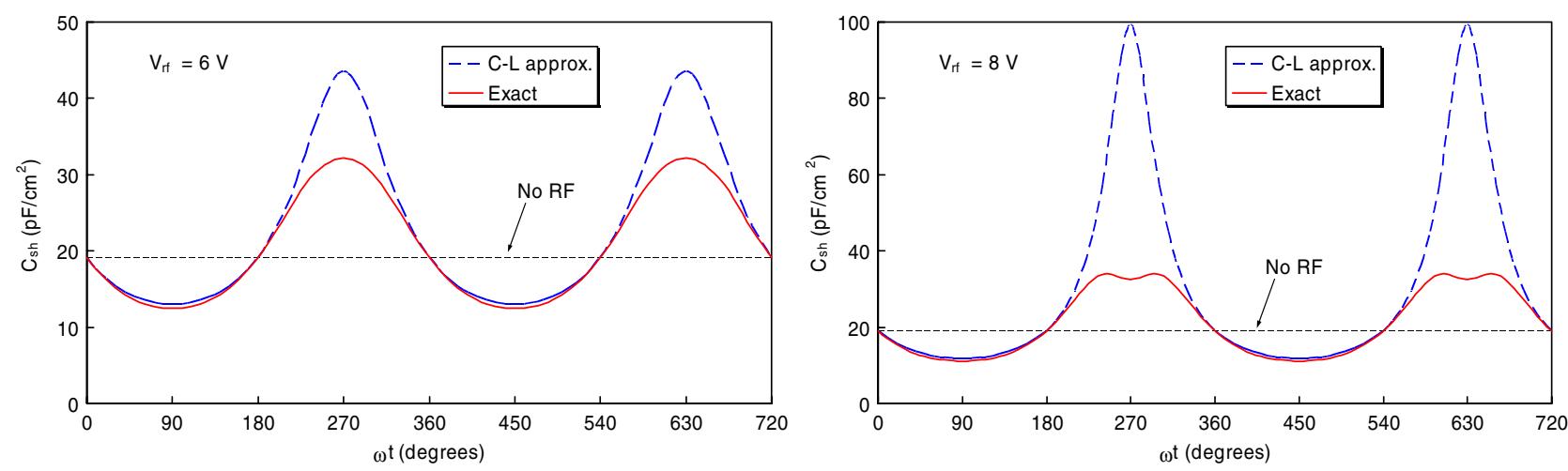

Figure 4. Variation of $C_{\mathrm{sh}}$ through two rf cycles for $V_{\mathrm{rf}}=6$ and $8 \mathrm{~V}$ (0-to-peak). The solid curve ( $\longrightarrow$ ) is the exact solution and the dashed curve (- - ) the C-L approximation. Parameters are $n=10^{12} \mathrm{~cm}^{-3}, K T_{\mathrm{e}}=3 \mathrm{eV}$ and $\eta_{\mathrm{dc}}=3$.

The minimum value of $\eta$ is thus given by equating these two fluxes:

$$
\begin{gathered}
\mathrm{e}^{\eta_{\min }}=\frac{A_{\mathrm{p}} n_{0} v_{\mathrm{r}}}{A_{\mathrm{w}} n_{\mathrm{s}} c_{\mathrm{s}}}=\frac{A_{\mathrm{p}}}{A_{\mathrm{w}}}\left(\frac{\mathrm{e}}{2 \pi} \frac{K T_{\mathrm{e}}}{m} \frac{M}{K T_{\mathrm{e}}}\right)^{1 / 2}, \\
\eta_{\min }=\frac{1}{2}\left[1+\ln \left(\frac{M}{2 \pi m} \frac{A_{\mathrm{p}}^{2}}{A_{\mathrm{w}}^{2}}\right)\right] .
\end{gathered}
$$

Note that 'e' here is not the unit charge ' $e$ '. From equation (33), $\bar{V}_{\text {s }}$ must rise to the value

$$
\bar{V}_{\mathrm{s}}=\bar{V}_{\mathrm{p}}-V_{\mathrm{rf}} \sin \omega t-\left(K T_{\mathrm{e}} / e\right) \eta_{\min } .
$$

For rf phases such that $\sin \omega t$ is negative and large enough that $\eta<\eta_{\min }$ in equation (33), the sheath capacitance is given by equation (32) with $\eta$ replaced by $\eta_{\min }$. Thus, for large $V_{\mathrm{rf}}, C_{\mathrm{sh}}$ obeys equation (32) for only part of the rf cycle. When $\sin \omega t$ swings sufficiently negative, $\bar{V}_{\mathrm{s}}$ rises to keep the plasma neutral, and $C_{\mathrm{sh}}$ remains constant during that part of the rf cycle.

Note, however, that equation (34) is valid only for $\eta \geqslant 0$. Electron saturation is reached at $\eta=0$, and $J_{\mathrm{e}}$ remains at the value $A_{\mathrm{p}} n_{0} v_{\mathrm{r}}$. That means that $\eta_{\min }$ must be non-negative and the argument of the logarithm in equation (37) must be $>1 / e$. If the probe area is so small that it can draw saturation electron current without raising $V_{\mathrm{s}}$, then the sheath vanishes, having infinite thickness. Indeed, if we replace $\eta$ by $\eta_{\min }=0$ in equation (32), we find that $C_{\mathrm{sh}}$ has the indeterminate form $0 / 0$, which can be seen to approach 0 by taking the derivatives of the numerator and denominator.

In summary, we see that $C_{\mathrm{sh}}$ varies in a very complicated way in the presence of $V_{\mathrm{rf}}$. There are four cases. (i) If $V_{\mathrm{rf}}$ is so small that $V_{\mathrm{s}}$ never oscillates below $\bar{V}_{\mathrm{p}}, C_{\mathrm{sh}}$ is affected only in higher order. (ii) For larger $V_{\mathrm{rf}}, C_{\mathrm{sh}}$ will vary nonsinusoidally with the phase of the rf. (iii) If $V_{\mathrm{rf}}$ is very large, $\bar{V}_{\mathrm{s}}$ will be changed by the probe current so as to keep $\eta$ positive, and $C_{\mathrm{sh}}$ will reach a limiting value during part of the $\mathrm{rf}$ cycle. (iv) If the probe is very small, $\eta_{\min }$ itself will saturate at the value 0 . These cases are illustrated in figures 4-6. Figure 4 compares $C_{\text {sh }}$ computed using equation (32) with that using equation (31). At $V_{\mathrm{rf}}=6 \mathrm{~V}$, the excursions are small enough that $\eta$ does not enter the region to the left of the peak in figure 2 and is near the limit of case (i). At $V_{\mathrm{rf}}=8 \mathrm{~V}$, that region is entered when $\sin \omega t \approx-1$, and the exact solution shows a dip in $C_{\mathrm{sh}}$. This dip reaches 0 at $V_{\mathrm{rf}}=9 \mathrm{~V}$, since $e\left(V_{\mathrm{s}}-V_{\mathrm{p}}\right)$ starts at $3 K T_{\mathrm{e}}=9 \mathrm{~V}$, and $V_{\text {rf }}$ is just sufficient to bring $V_{\mathrm{s}}$ down to $V_{\mathrm{p}}$ at its extremum. This is case (ii).

Figure 5 shows case (iii), when $\eta$ is limited by $\eta_{\min }$, but $\eta_{\min }$ is still above the dc value of $\eta=3$. The 'exact' solution of equation (32) is compared with that when the $\eta_{\min }$ cutoff is imposed by the fact that $V_{\mathrm{s}}$ is dragged upwards by the probe current. This rare case happens only when the plasma chamber is small, so that the ratio $A_{\mathrm{w}} / A_{\mathrm{p}}$ in equation (37) is not very large. However, we have observed experimentally that the shift in $\bar{V}_{\mathrm{s}}$ is larger than expected from the calculation above, so that case (iii) may occur for larger values of $A_{\mathrm{w}} / A_{\mathrm{p}}$.

Figure 6 shows case (iv) when $A_{\mathrm{w}} / A_{\mathrm{p}}$ has a more normal value of $2800 / 0.047$ (an $0.015 \times 1 \mathrm{~cm}$ diam probe in a $30 \mathrm{~cm}$ diam $\times 30 \mathrm{~cm}$ high chamber). In this case, the large $V_{\mathrm{s}}$ oscillations quickly bring $V_{\mathrm{s}}$ down to $V_{\mathrm{p}}$ whenever $\sin \omega t$ is negative, and $\eta_{\min } \rightarrow 0$ for a large portion of the rf period. $C_{\mathrm{sh}}$ also goes to 0 , corresponding to the left edge of the exact curve in figure 2 . The only difference between the curves is that the 'exact' equation (32) has no solution for $\eta<0$, while 'cutoff' solution replaces $\eta$ with $\eta_{\min }=0$.

The sudden jumps in $C_{\mathrm{sh}}$ would give rise to high harmonics in the probe current. These jumps are an artefact of the planegeometry idealization. In cylindrical geometry the electron current does not saturate abruptly but slowly grows as the electron sheath expands. The curves in figure 6 should be smoothed out, but we shall see in section 5 that the situation is not simple. In the next two sections, we examine methods devised to avoid the complicated behaviour of $C_{\mathrm{sh}}$ by limiting the effective $V_{\text {rf }}$ to small values.

\subsection{Partially compensated probe}

The most common way to deal with rf fluctuations in $V_{0}$ is to use tuned inductors and auxiliary floating electrodes, a method tried by Gagne and Cantin [8,9] and further developed by Godyak et al [10], Sudit and Chen [6] and Mahony et al [11]. A partially compensated probe is shown in figure 7 . The probe tip is located at P. The sheath capacitance $C_{\text {sh }}$ connects it to the space potential $V_{\mathrm{s}}$. A choke chain with impedance $Z_{\mathrm{ck}}$ consisting of inductors and their stray capacitances filters out the rf fluctuations $\tilde{V}_{\mathrm{s}}$ from the resistor $R_{\mathrm{m}}$ across which the probe current is measured. The value of $R_{\mathrm{m}}$ is small and can be neglected in this discussion. Ideally, the probe tip then 

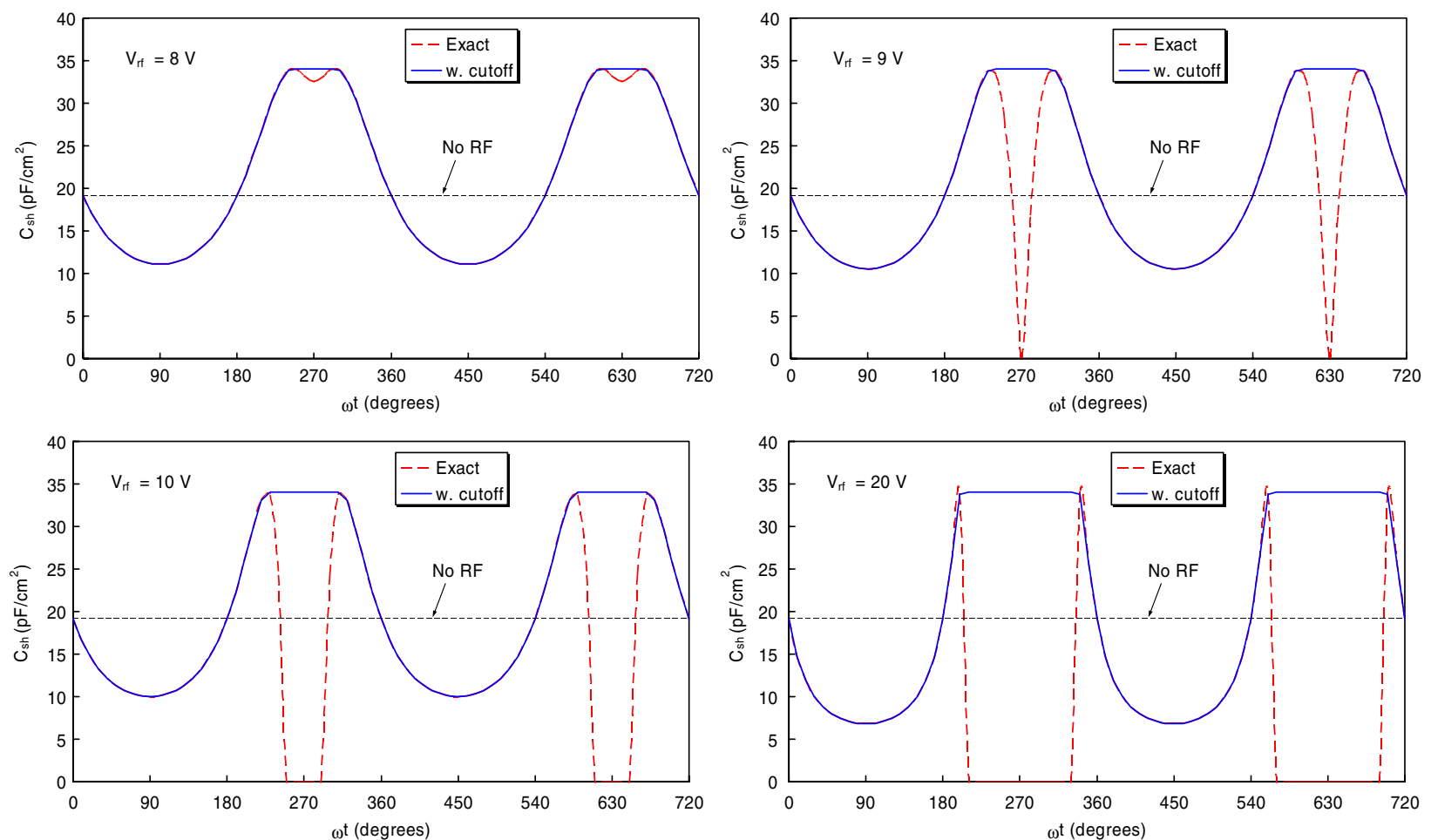

Figure 5. Comparison of the 'exact' solution (- - -) with that imposed by $\eta_{\min }$ as $V_{\mathrm{rf}}$ is increased $(-)$. The $V_{\mathrm{rf}}=8 \mathrm{~V}$ case is the same as in figure 4 . The ratio $A_{\mathrm{w}} / A_{\mathrm{p}}$ is only 100 here.
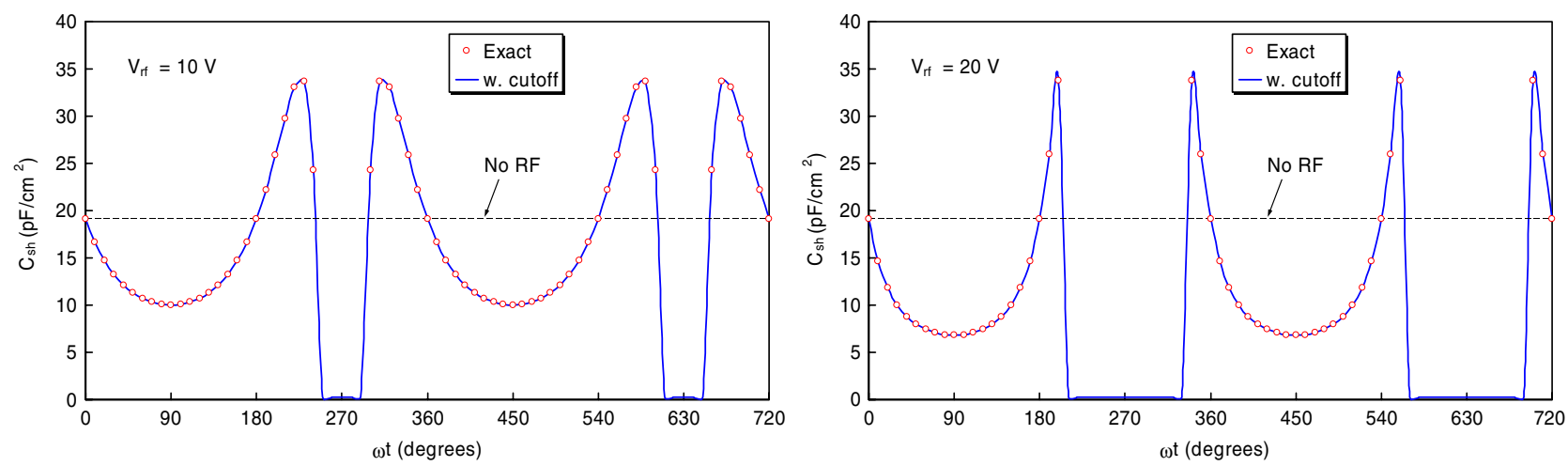

Figure 6. Behaviour of $C_{\mathrm{sh}}$ for small probes and large $V_{\mathrm{rf}}$. The 'exact' solution (O) does not exist where the 'cutoff' solution ( $\longrightarrow$ ) gives $C_{\mathrm{sh}}=0$.

fluctuates with $\tilde{V}_{\text {s }}$ so that the applied dc voltage $V_{\mathrm{p}}$ is the only voltage between the probe and the plasma. Ignoring the stray capacitance $C_{\mathrm{s}}$ for the moment, we see that $C_{\mathrm{sh}}$ and $Z_{\mathrm{ck}}$ form a voltage divider, and the rf signal at $\mathrm{P}$ is

$$
\tilde{V}_{\mathrm{p}}=\tilde{V}_{\mathrm{s}} \frac{Z_{\mathrm{ck}}}{Z_{\mathrm{ck}}+Z_{\mathrm{sh}}}
$$

where $Z_{\mathrm{sh}}$ is $-j / \omega C_{\mathrm{sh}}$. To suppress rf pickup, therefore, $\left|Z_{\mathrm{ck}}\right|$ must be much larger than $\left|Z_{\mathrm{sh}}\right|$ so that $\tilde{V}_{\mathrm{p}}$ follows $\tilde{V}_{\mathrm{s}}$ closely. To get probe $I-V$ characteristics unaffected by $\tilde{V}_{\mathrm{s}}$ requires

$$
\eta_{\mathrm{rf}}=\left(e / K T_{\mathrm{e}}\right)\left(\tilde{V}_{\mathrm{s}}-\tilde{V}_{\mathrm{p}}\right) \ll 1,
$$

where

$$
\eta=\eta_{\mathrm{dc}}+\eta_{\mathrm{rf}}=\left(e / K T_{\mathrm{e}}\right)\left(\bar{V}_{\mathrm{s}}+\tilde{V}_{\mathrm{s}}-\bar{V}_{\mathrm{p}}-\tilde{V}_{\mathrm{p}}\right) .
$$

From equation (39) with $\tilde{V}_{\mathrm{s}} \approx \tilde{V}_{\text {rf }}$, the requirement is [7]

$$
\frac{e \tilde{V}_{\mathrm{rf}}}{K T_{\mathrm{e}}}\left|\frac{Z_{\mathrm{sh}}}{Z_{\mathrm{ck}}+Z_{\mathrm{sh}}}\right| \approx \frac{e \tilde{V}_{\mathrm{rf}}}{K T_{\mathrm{e}}}\left|\frac{Z_{\mathrm{sh}}}{Z_{\mathrm{ck}}}\right| \ll 1 .
$$

To get an order of magnitude, we can use equation (29) to estimate $C_{\mathrm{sh}}$. For typical parameters $A_{\mathrm{p}}=0.047 \mathrm{~cm}^{2}$ ( $0.15 \mathrm{~mm}$ diam $\times 1 \mathrm{~cm}$ long), $K T_{\mathrm{e}}=3 \mathrm{eV}, n=10^{12} \mathrm{~cm}^{-3}$, this gives $C_{0}=42 \mathrm{pF}$ and $\left|Z_{\mathrm{sh}}\right|=280 \Omega$ at $13.56 \mathrm{MHz}$. Here we have assumed $\bar{V}_{\mathrm{p}}$ near floating potential, so that $\bar{V}_{\mathrm{p}}-\bar{V}_{\mathrm{s}} \approx 5$. If $\tilde{V}_{\text {rf }}=100 \mathrm{~V}$, we would require $\left|Z_{\mathrm{ck}}\right| \gg 10 \mathrm{k} \Omega$, or $\left|Z_{\mathrm{ck}}\right| \geqslant 100 \mathrm{k} \Omega$. At lower densities, this value increases as $n^{-\frac{1}{2}}$. To get an impedance this high usually requires using tuned chokes whose self-resonance is at the rf frequency. 


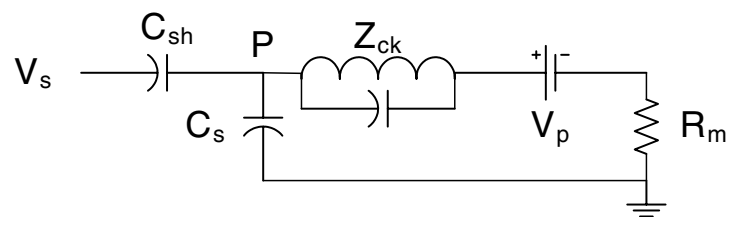

Figure 7. Isolation of a probe with a choke.

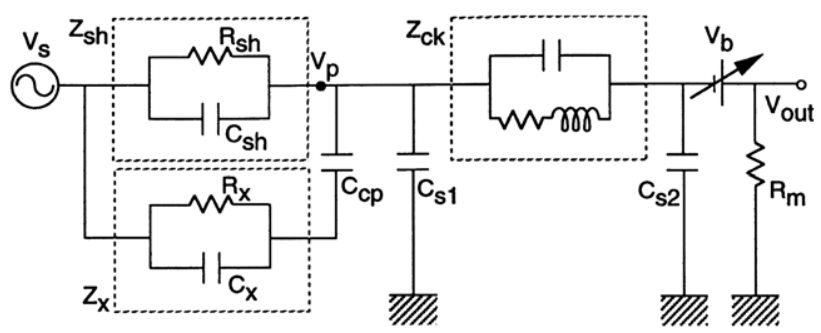

Figure 8. (from [1])

\subsection{Fully compensated probe}

A choke chain alone, however, is insufficient because of the stray capacitance $C_{\mathrm{s}}$ of the short wire between the probe tip and the choke chain [6,7]. If $C_{\mathrm{s}}$ connects $\mathrm{P}$ to ground, it effectively decreases the value of $\left|Z_{\mathrm{sh}}\right|$. If $C_{\mathrm{s}}$ connects $\mathrm{P}$ to the plasma through a ceramic probe insulator, it has little effect, since it simply adds a small amount to $C_{\mathrm{sh}}$. If $\tilde{V}_{\mathrm{s}}$ varies in space, there may be a small difference between the $\tilde{V}_{\mathrm{s}}$ sampled by $C_{\mathrm{s}}$ and that seen by the probe tip, but this cannot be a large effect.

However, in a real situation, it was found [6] that $\left|Z_{\mathrm{c}}\right| \approx$ $200 \mathrm{k} \Omega$ gave insufficient $\mathrm{rf}$ isolation even if a metal probe shaft was not used. In this case, an auxiliary electrode or 'compensation electrode' of large area $A_{\mathrm{x}}$ (and hence large $C_{\mathrm{x}}$ ) is required to detect the rf changes in $V_{0}$. Figure 8 shows the probe circuit with both sheaths included [6]. Here $C_{\text {sh }}$ is on the probe tip and $C_{\mathrm{x}}$ is on the auxiliary electrode. The latter is coupled to the probe through the relatively large capacitor $C_{\mathrm{cp}} . C_{\mathrm{x}}$ is given by equations (32) and (33) but with a larger area $A_{\mathrm{x}}$, large enough to satisfy equation (42) even if $Z_{\mathrm{ck}}$ is bypassed by the stray capacitance $C_{\mathrm{s} 1}$.

As far as the probe is concerned, $\tilde{V}_{\mathrm{p}}$ is driven by the compensation electrode and is given by equation (39) with $Z_{\mathrm{x}}$ in place of $Z_{\mathrm{sh}}$ :

$$
\tilde{V}_{\mathrm{p}}=\tilde{V}_{\mathrm{s}}\left|\frac{Z_{\mathrm{ck}}}{Z_{\mathrm{ck}}+Z_{\mathrm{x}}}\right| .
$$

The requirement of equation (42) is then relaxed to

$$
\frac{e \tilde{V}_{\mathrm{rf}}}{K T_{\mathrm{e}}}\left|\frac{Z_{\mathrm{x}}}{Z_{\mathrm{ck}}+Z_{\mathrm{x}}}\right| \approx \frac{e \tilde{V}_{\mathrm{rf}}}{K T_{\mathrm{e}}}\left|\frac{Z_{\mathrm{x}}}{Z_{\mathrm{ck}}}\right| \ll 1,
$$

and $\left|Z_{\mathrm{ck}}\right|$ can be smaller by the ratio $A_{\mathrm{x}} / A_{\mathrm{p}}$. If $A_{\mathrm{x}}$ is large enough, it may not be necessary to use resonant chokes. For instance, if $A_{\mathrm{p}} \approx .05 \mathrm{~cm}^{2}$, and $A_{\mathrm{x}} \approx 5 \mathrm{~cm}^{2}(0.5 \mathrm{~cm} \mathrm{diam}$ $\times 3 \mathrm{~cm}$ long), $\left|Z_{\mathrm{ck}}\right|$ can be reduced a factor 100 below the $100 \mathrm{k} \Omega$ calculated in the previous example. However, note that $C_{\text {sh }}$ depends on $1 / \lambda_{\mathrm{D}} \propto \sqrt{ } n$. If $n \approx 10^{9} \mathrm{~cm}^{-3}$ instead of $10^{12} \mathrm{~cm}^{-3},\left|Z_{\mathrm{sh}}\right|$ is increased by a factor of 32 , and $\left|Z_{\mathrm{ck}}\right|$ of order $30 \mathrm{k} \Omega$ is still required.

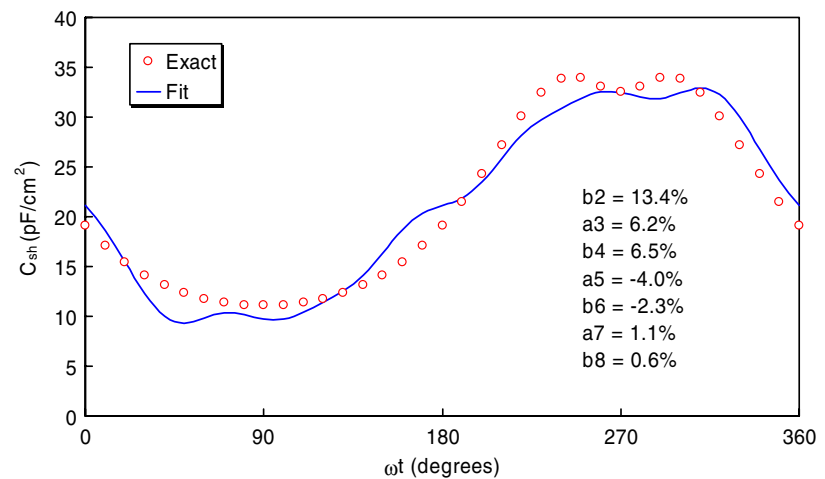

Figure 9. A Fourier fit to the exact curve of figure 4 for $V_{\mathrm{rf}}=8 \mathrm{~V}$. The harmonic amplitudes relative to the fundamental are shown.

\subsection{Generation of harmonics}

Since $C_{\mathrm{sh}}$ varies with $V_{\mathrm{s}}$, the rf probe current will be nonsinusoidal, and harmonics of the rf frequency will be generated. To estimate this, we use the approximate formula (31):

$$
C_{\mathrm{sh}}=C_{0}\left(1+\frac{V_{\mathrm{rf}} \sin \omega t}{\bar{V}_{\mathrm{s}}-\bar{V}_{\mathrm{p}}}\right) .
$$

The ac electron current to the probe is given by

$$
\tilde{I}_{\mathrm{e}}=C_{\mathrm{sh}}\left(\mathrm{d} V_{\mathrm{s}} / \mathrm{d} t\right) .
$$

From equations (30) and (45), we obtain

$$
\tilde{I}_{\mathrm{e}}=C_{0}\left(1+\frac{V_{\mathrm{rf}} \sin \omega t}{\bar{V}_{\mathrm{s}}-\bar{V}_{\mathrm{p}}}\right)^{-3 / 4}\left(V_{\mathrm{rf}} \omega \cos \omega t\right) .
$$

For sufficiently small $V_{\text {rf }}$, Taylor expansion gives

$$
\begin{aligned}
\tilde{I}_{\mathrm{e}} & =C_{0}\left(1-\frac{3}{4} \frac{V_{\mathrm{rf}} \sin \omega t}{\bar{V}_{\mathrm{s}}-\bar{V}_{\mathrm{p}}}\right)\left(V_{\mathrm{rf}} \omega \cos \omega t\right) \\
& =\omega C_{0} V_{\mathrm{rf}}\left(\cos \omega t-\frac{3}{8} \frac{V_{\mathrm{rf}} \sin 2 \omega t}{\bar{V}_{\mathrm{s}}-\bar{V}_{\mathrm{p}}}\right) .
\end{aligned}
$$

When the expansion is valid, the 2 nd harmonic is smaller than the fundamental by a factor [6]

$$
\frac{3}{8} \frac{V_{\mathrm{rf}}}{\bar{V}_{\mathrm{s}}-\bar{V}_{\mathrm{p}}}
$$

This is, of course, valid only for very small values of $V_{\mathrm{rf}}$. To see the effect of a more exact calculation, we have Fourier analysed the fairly smooth curve of figure $4(b)$, representing a $V_{\text {rf }}$ of only $8 \mathrm{~V}$. The Fourier fit up to the 8 th harmonic is shown in figure 9. The non-zero coefficients $a_{\mathrm{n}}$ of $\sin (n \omega t)$ and $b_{\mathrm{n}}$ of $\cos (n \omega t)$ are given relative to that of the fundamental, $a_{1}$. The largest harmonic, the 2nd, has $13 \%$ amplitude in this case. The leading term in $\tilde{I}_{\mathrm{e}}$ is still given by equation (48).

\subsection{The sheath resistance}

The real part of $Z_{\mathrm{sh}}$, corresponding to the particle current to the probe, is also nonlinear. We consider only the electron current, since the ion current is comparatively constant and is 
not greatly changed by fluctuations in $V_{\mathrm{s}}$. For a Maxwellian distribution the electron current is given by equation (34):

$$
I_{\mathrm{e}}=-e A_{\mathrm{p}} n_{0} v_{\mathrm{r}} \mathrm{e}^{e\left(V_{\mathrm{s}}-V_{\mathrm{p}}\right) / K T_{\mathrm{e}}} .
$$

The sheath resistance is defined by

$$
R_{\mathrm{sh}}=\mathrm{d} V / \mathrm{d} I \text {, }
$$

so that, in the absence of $\mathrm{rf}$, the dc resistance is given by

$$
R_{\mathrm{sh}}^{-1}=\mathrm{d} I_{\mathrm{e}} / \mathrm{d}\left(V_{\mathrm{p}}\right)=\left(e / K T_{\mathrm{e}}\right)\left|I_{\mathrm{e}}\right|,
$$

with $V_{\mathrm{s}}=\bar{V}_{\mathrm{s}}=$ constant. At the floating potential, $\left|I_{\mathrm{e}}\right|$ is equal to $I_{\mathrm{i}}$, and $R_{\text {sh }}$ there is given by

$$
\begin{array}{r}
R_{\mathrm{sh}}\left(V_{f}\right)=\frac{K T_{\mathrm{e}}}{e} \frac{1}{e I_{i}}=\frac{K T_{\mathrm{e}}}{e^{2}} \frac{1}{n_{\mathrm{s}} c_{\mathrm{s}} A_{\mathrm{p}}} \\
=\frac{K T_{\mathrm{e}}}{e^{2}} \frac{1}{\mathrm{e}^{-\frac{1}{2}} n_{0} c_{\mathrm{s}} A_{\mathrm{p}}}=\frac{\left(e M K T_{\mathrm{e}}\right)^{\frac{1}{2}}}{A_{\mathrm{p}} n_{0} e^{2}} .
\end{array}
$$

Note that the 'e' not in italics is not the unit charge. As the probe bias $\bar{V}_{\mathrm{p}}$ increases, $\left|I_{\mathrm{e}}\right|$ increases, and $R_{\mathrm{sh}}$ decreases from this value exponentially.

In the presence of $\mathrm{rf}, V_{\mathrm{s}}$ will oscillate and be given by $V_{\mathrm{s}}=\bar{V}_{\mathrm{s}}+V_{\mathrm{rf}} \sin \omega t$. If the probe is uncompensated, $V_{\mathrm{p}}$ will remain at $V_{\mathrm{p}}$. The $I-V$ curve will oscillate horizontally, and its slope will change, causing $R_{\mathrm{sh}}$ to change nonlinearly during the rf cycle. Its value is still given by equations (52) and (50) if $V_{\mathrm{s}}$ is given its unsteady value. If the probe is rf compensated, as subsection 4.4, $V_{\mathrm{p}}$ will follow $V_{\mathrm{s}}$, and the slope of the $I-V$ curve will not change as much. $R_{\mathrm{sh}}$ is still given by equations (52) and (50) if $V_{\mathrm{s}}-V_{\mathrm{p}}$ is given its compensated value. From equation (43) we obtain

$$
\tilde{V}_{\mathrm{s}}-\tilde{V}_{\mathrm{p}}=V_{\mathrm{rf}} \sin \omega t\left|\frac{Z_{\mathrm{x}}}{Z_{\mathrm{ch}}+Z_{\mathrm{x}}}\right| .
$$

The instantaneous sheath resistance from equations (52), (50) and (54) can now be written as

$$
R_{\mathrm{sh}}=\frac{K T_{\mathrm{e}}}{e} \frac{\mathrm{e}^{-\left[\bar{V}_{\mathrm{s}}-\bar{V}_{\mathrm{p}}+\frac{e}{K T_{\mathrm{e}}}\left|\frac{Z_{\mathrm{x}}}{Z_{\mathrm{ch}}+Z_{\mathrm{x}}}\right| V_{\mathrm{rf}} \sin \omega t\right]}}{e A_{\mathrm{p}} n_{0} v_{\mathrm{r}}} .
$$

The same proviso on negative values of $\sin \omega t$ that makes the exponent positive applies here, but with good compensation this should not happen. The dynamic impedance of a plane sheath in a strong rf environment is then given by

$$
Z_{\mathrm{sh}}=R_{\mathrm{ch}}-j / \omega C_{\mathrm{sh}},
$$

in which the resistive and reactive elements have been treated in detail above.

\section{Cylindrical geometry}

A compensation electrode is usually large enough compared with $\lambda_{\mathrm{D}}$ that the sheath on it can be treated as planar. A wire probe tip, however, is likely to be made with radius $R_{\mathrm{p}}$ smaller than or comparable to $\lambda_{\mathrm{D}}$ in order for the orbitalmotion-limited (OML) probe theory to be applicable. In that case, the probe sheath has to be treated in cylindrical geometry. In the electron retardation region, Poisson's equation (11) is replaced by the cylindrical version [12] of the Allen-BoydReynolds [13] (ABR) equation

$$
\frac{1}{\rho} \frac{\mathrm{d}}{\mathrm{d} \rho}\left(\rho \frac{\mathrm{d} \eta}{\mathrm{d} \rho}\right)=\frac{J}{\rho} \eta^{-\frac{1}{2}}-\mathrm{e}^{-\eta}, \quad J \equiv \frac{1}{2 \pi \sqrt{2}} \frac{I_{\mathrm{i}}}{e n_{0}} \frac{1}{\lambda_{\mathrm{D}} c_{\mathrm{S}}},
$$

where $\rho=r / \lambda_{\mathrm{D}}$. This equation is not amenable to an analytic solution suitable for probe design. It has to be integrated from infinity, and it does not make sense [14] to define a sheath edge where $v_{\mathrm{i}}=c_{\mathrm{s}}$. Furthermore, the ion current $I_{\mathrm{i}}$ has to be assumed at the outset.

Although we cannot easily extend the plane results for the retardation region to cylinders, we can treat the region of electron saturation. When $V_{\mathrm{p}}>V_{\mathrm{s}}$ so that $\eta<0$, electrons are accelerated towards the probe. Their thermal velocities will cause those with high angular momentum to orbit the probe and miss it; they cannot be treated as a cold fluid, as we did with the ions. The equations have been solved numerically by Laframboise [15], but these specific results cannot be applied to the general case. However, if $R_{\mathrm{p}} / \lambda_{\mathrm{D}}$ is small enough, it is possible to use the convenient OML theory of Mott-Smith and Langmuir [16], as summarized by Chen [17]. The orbits of particles spiralling in to an attractive probe are calculated using energy and momentum conservation as in subsection 2.1, but in the case of electrons one cannot neglect their energy spread at the sheath edge. For a Maxwellian distribution at $r=s$, the OML result for saturation electron current is

$$
I_{\mathrm{e}, \mathrm{sat}}=A_{\mathrm{p}} e n_{0} v_{\mathrm{r}} F,
$$

where $v_{\mathrm{r}}$ is given by equation (34) and $F$ is the function

$$
F \equiv \frac{1}{\varepsilon} \operatorname{erf}\left(\Phi^{\frac{1}{2}}\right)+\mathrm{e}^{-\eta}\left[1-\operatorname{erf}(\Phi-\eta)^{\frac{1}{2}}\right]
$$

in which

$$
\begin{aligned}
& \varepsilon \equiv R_{\mathrm{p}} / s \quad<1, \quad \Phi \equiv \eta /\left(1-\varepsilon^{-2}\right), \\
& \Phi-\eta=\eta /\left(\varepsilon^{2}-1\right) .
\end{aligned}
$$

Here $\eta$ is still defined by equation (7) and is negative for $V_{\mathrm{p}}>$ $V_{\mathrm{s}}$, so that the arguments of the error functions

$$
\operatorname{erf}(x) \equiv \int_{0}^{x} \mathrm{e}^{-t^{2}} \mathrm{~d} t \rightarrow[x \rightarrow 0] \frac{2 x}{\sqrt{\pi}}
$$

are real. The sheath radius $s$ has to be arbitrarily assumed, since there is no Bohm criterion for electrons when $T_{\mathrm{e}}>T_{\mathrm{i}}$. However, it turns out that $F$ is extremely insensitive to $\varepsilon$ for all $\varepsilon>10$, a fact that Langmuir could not point out because he did not have personal computers. We may therefore take the $s \rightarrow \infty$ limit of equation (59), obtaining

$$
F(\eta)=\frac{2}{\sqrt{\pi}}(-\eta)^{\frac{1}{2}}+\mathrm{e}^{-\eta}\left[1-\operatorname{erf}(-\eta)^{\frac{1}{2}}\right] .
$$

Note that at the space potential $\eta=0, F \rightarrow 1$ and $F^{\prime}(\eta) \rightarrow$ -1 , so that $I_{\mathrm{e}}$,sat joins smoothly onto the transition region (equation (34)). An ideal OML probe curve is shown in figure 10. At $V_{\mathrm{s}}=0$, the junction at $V_{\mathrm{s}}$ is smooth for a cylindrical probe but abrupt for a plane probe. As $V_{\text {rf }}$ oscillates with amplitude $6 \mathrm{~V}$, the probe bias $V_{\mathrm{p}}$ effectively oscillates relative to this curve between the limits shown. In this case, $V_{\mathrm{rf}}$ is large enough that the probe enters the electron saturation 


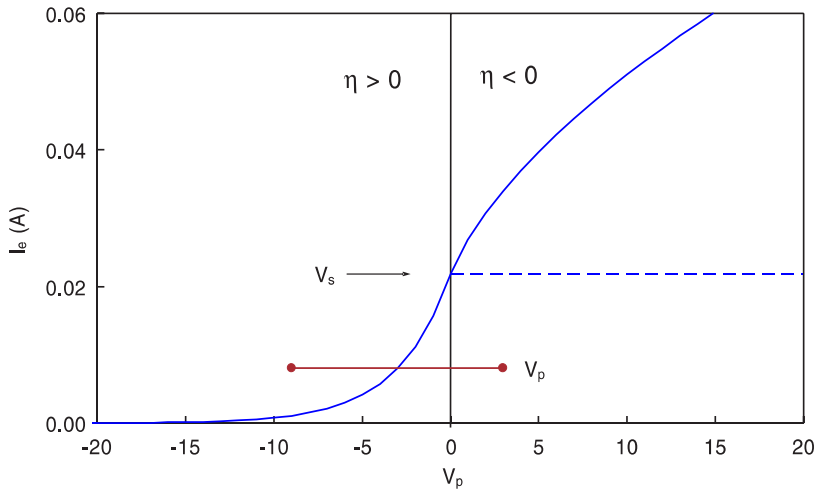

Figure 10. An ideal OML curve for a cylindrical probe with $R_{\mathrm{p}}=0.0075$ and $L=1 \mathrm{~cm}$. The argon plasma has $K T_{\mathrm{e}}=3 \mathrm{eV}$ and $n=10^{11} \mathrm{~cm}^{-3}$. The dashed line is the saturation electron current for a plane probe. The effective excursion of $V_{\mathrm{p}}$ is shown for $V_{\mathrm{rf}}=6 \mathrm{~V}$ and $\eta_{0}=1$.

region during part of the rf cycle, but $I_{\mathrm{e}}$ does not change discontinuously, as it does in the plane case.

Equations (58) and (62) give $I_{\mathrm{e}}$ without the need for solution of Poisson's equation to get $V(r)$. Whether a collisionless electron coming from infinity hits the probe or not depends only on its initial energy and angular momentum regardless of the shape of $V(r)$. The requirement $R_{\mathrm{p}} \ll \lambda_{\mathrm{D}}$ stems from the fact that $n$ has to be small enough that there is no absorption radius $R_{\mathrm{a}}$ inside of which all electrons are collected, thus increasing the effective probe radius from $R_{\mathrm{p}}$ to $R_{\mathrm{a}}$. To calculate $C_{\mathrm{sh}}$, however, equation (21) requires a knowledge of $\eta(\rho)$, which is not available from the OML theory. Note, however, that the solution given by equations (58) and (62) is self-similar; the only scalelength is given by $R_{\mathrm{p}}$. The ratio $I_{\mathrm{e}} /$ $R_{\mathrm{p}}$ depends only on $\eta$. Thus, as $R_{\mathrm{p}}$ changes, the picture is the same, and only the scale changes. All the particle trajectories have the same shape, and therefore the surface charge density $\rho_{\mathrm{s}}$ is proportional to $I_{\mathrm{e}} / R_{\mathrm{p}}$, and hence to $F(\eta)$. Let

$$
\rho_{\mathrm{s}}=\tau_{1}\left(I_{\mathrm{e}} / A_{\mathrm{p}}\right),
$$

where $\tau_{1}$ is a constant with the dimensions of time. Equations (20), (58) and (63) then give

$$
\frac{C_{\mathrm{sh}}}{A_{\mathrm{p}}}=\frac{\mathrm{d} \rho_{\mathrm{s}}}{\mathrm{d} V}=-\frac{e}{K T_{\mathrm{e}}} \frac{\mathrm{d}}{\mathrm{d} \eta}\left(\tau_{1} n_{0} e v_{\mathrm{r}} F\right) .
$$

With equations (9) and (34), this can be written as

$$
\frac{C_{\mathrm{sh}}}{A_{\mathrm{p}}}=-\frac{1}{\sqrt{2 \pi}} \frac{\varepsilon_{0}}{\lambda_{\mathrm{D}}} \omega_{\mathrm{p}} \tau_{1} F^{\prime}(\eta) .
$$

Differentiating equation (62) gives

$$
F^{\prime}(\eta)=-\mathrm{e}^{-\eta}\left\{1-\operatorname{erf}\left[(-\eta)^{\frac{1}{2}}\right]\right\},
$$

where $\eta$ is negative. The sheath capacitance in electron saturation is therefore

$$
\frac{C_{\mathrm{sh}}}{A_{\mathrm{p}}}=\frac{1}{\sqrt{2 \pi}} \frac{\varepsilon_{0}}{\lambda_{\mathrm{D}}} \omega_{\mathrm{p}} \tau_{1} \mathrm{e}^{-\eta}\left\{1-\operatorname{erf}\left[(-\eta)^{\frac{1}{2}}\right]\right\} .
$$

One might think that the unknown constant $\tau_{1}$ can be evaluated by matching smoothly to the solution for $\eta>0$, but this is not

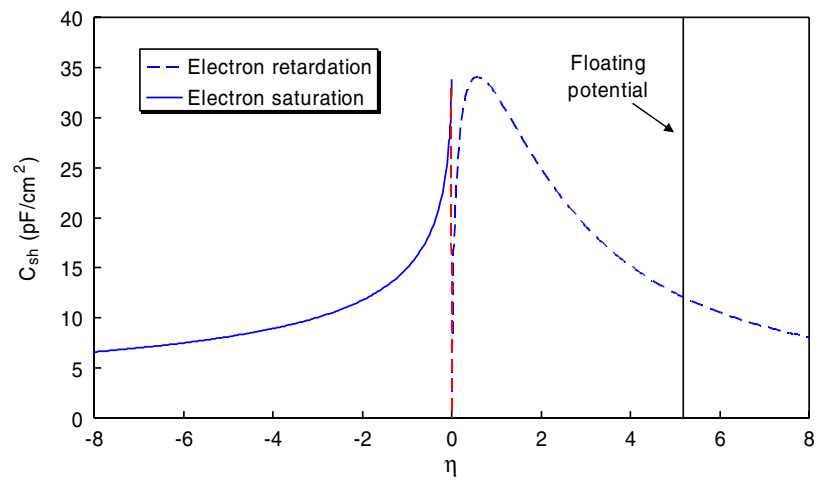

Figure 11. Behaviour of $C_{\mathrm{sh}}$ of a cylindrical electron sheath ( - ); the magnitude is approximate. Also shown for comparison is $C_{\mathrm{sh}}$ for a plane ion sheath (- - - ) from figure 2. Both curves dip to 0 at the space potential.

the case. In the limit $\eta \rightarrow 0$,

$$
\frac{C_{\mathrm{sh}}}{A_{\mathrm{p}}}=\frac{1}{\sqrt{2 \pi}} \frac{\varepsilon_{0}}{\lambda_{\mathrm{D}}} \omega_{\mathrm{p}} \tau_{1} \neq 0,
$$

whereas $C_{\mathrm{sh}}$ at the space potential has to vanish because there is no sheath. Actually, $C_{\mathrm{sh}}$ has to drop to zero even from the electron saturation side. If $T_{\mathrm{i}}$ is finite, though very small, it begins to be collected when $-\eta$ is small, and equation (67) is no longer valid. $C_{\mathrm{sh}}$ will fall to zero as in figure 2 but much more steeply, in a voltage range scaled to $T_{\mathrm{i}}$ rather then $T_{\mathrm{e}}$. Although the absolute magnitude of $C_{\mathrm{sh}}$ is not known, we can show its behaviour according to equation (67) in figure 11. There is still a discontinuity at $\eta=0$, but it is so spiky that it will not be seen, and $C_{\mathrm{sh}}$ will vary much more smoothly than in figure 5 when $V_{\text {rf }}$ brings $\eta$ into the electron saturation region.

The statement that the sheath vanishes at $V_{\mathrm{p}}=V_{\mathrm{s}}$ does not hold when there is a dc magnetic field. The probe then casts a shadow, and particles are depleted from the tube of force intercepted by the probe. The space potential inside the tube is different from that outside, and it varies in a way that depends on the diffusion, classical or anomalous, of electrons across the B-field into the tube. Since $V_{\mathrm{p}}$ cannot be $V_{\mathrm{s}}$ everywhere, there is always going to be a sheath, and $C_{\mathrm{sh}}$ cannot vanish as it does for an infinitesimal probe in a B-field-free plasma.

A solution for $C_{\mathrm{sh}}$ in the Child-Langmuir approximation for cylinders $(\eta \gg 1)$ is also available [18], but the series solution is quite cumbersome. For emission from a thin wire out to a sheath edge, Langmuir [18] showed that the solution is insensitive to $s / r$ for $s / r>10$. This may not be true for ion emission from the outer cylinder rather than the inner one. In any case this $\mathrm{C}-\mathrm{L}$ solution cannot be connected to the electron retardation region without numerical integration.

\section{Conclusion}

Both the real and imaginary parts of the sheath impedance of a Langmuir probe vary nonlinearly with rf fluctuations in space potential. An equation for the time-dependent sheath capacitance in plane geometry is derived including both the Child-Langmuir and Debye sheaths. Inclusion of the Debye sheath leads to violent oscillations of the sheath capacitance 
which may lead to generation of many harmonics of the rf frequency. For a cylindrical probe the sheath capacitance is given for the electron saturation region, but the transition region requires numerical integration. The effect of $\mathrm{rf}$ on probe characteristics can be minimized with an appropriate compensation circuit, whose parameters are specified.

\section{Acknowledgment}

This work was partially supported by the National Science Foundation Grant No DMI-0115570.

\section{References}

[1] Lieberman M A 1988 IEEE Trans. Plasma Sci. 16638

[2] Lieberman M A 1989 IEEE Trans. Plasma Sci. 17338

[3] Godyak V A and Sternberg N 1991 Proc. 20th Int. Conf. on Phenomena in Ionized Gases (Barga, Italy, July 8-12, 1991) p 661

[4] Godyak V A and Sternberg N 1990 Phys. Rev. A 422299
[5] Zhang Y, Liu J, Liu Y, and Wang X 2004 Phys. Plasmas 113840

[6] Sudit I D and Chen F F 1994 Plasma Sources Sci. Technol. 3162

[7] Godyak V A and Piejak R B 1990 J. Appl. Phys. 683157

[8] Gagné R R J and Cantin A 1972 J. Appl. Phys. 432639

[9] Cantin A and Gagné R R J 1977 Appl. Phys. Lett. 30316

[10] Godyak V A, Piejak R B and Alexandrovich B M 1992 Plasma Sources Sci. Technol. 136

[11] Mahony C M O, Maguire P D and Graham W G 2005 Plasma Sources Sci. Technol. 14 S60

[12] Chen F F 1965 Plasma Phys. 747

[13] Allen J E, Boyd R L F and Reynolds P 1957 Proc. Phys. Soc. (Lond.) B 70 297

[14] Chen F F and Arnush D 2001 Phys. Plasmas 85051

[15] Laframboise J G 1966 University of Toronto Institute of Aerospace Studies Report No 100, National Technical Information Service Document No. AD634596

[16] Mott-Smith H M and Langmuir I 1926 Phys. Rev. 28727

[17] Chen F F 1965 Electric probes Plasma Diagnostic Techniques ed R H Huddlestone and S L Leonard (New York: Academic) Chapter 4, pp 128-35

[18] Langmuir I 1913 Phys. Rev. 2450 